\title{
Le insegne ed i riti d'ascesa degli imperatori. Senso e significatività dei segni dell'eccellenza da Costantino a Valentiniano I
}

The Insignia and the Election Rite of the Emperors. Sense and Significance of the Excellence Signs from Constantine to Valentinian I

Antonio Pio Di Cosmo

Pontificio Istituto Orientale, Roma, Italia

apiocosmo@outlook.it

Recibido: 26-01-2020. Aceptado: 05-03-2020.

\section{Riassunto}

Il presente contributo analizza il ruolo giocato dai segni del potere imperiale ed in particolare dalle insegne nei riti di elezione. Questa ricerca applica le conoscenze in materia archeologica, antropologica e storica. Si racconta il rituale di elezione e si risolvono le questioni circa i problemi di rappresentazione dell'augusto nelle cerimonie d'ascesa. In questo modo si vagliano le strategie di comunicazione che modellano i protocolli di creazione degli augusti.

\begin{abstract}
This contribution analyses the role played by the symbols of status, in particular the function of the imperial outfit, in the election ceremony through the use of archaeological, anthropological and historical evidence. It considers the election ritual and addresses a number of questions related to the problems of representation of Augusti during these ceremonies. Hence, the paper examines the communication strategies that shaped the protocols during the elections.
\end{abstract}

\section{Introduzione}

La presente indagine è volta ad ottenere una più profonda comprensione della funzionalità delle insegne imperiali svolta nel marco delle strategie di autorappresentazione di un'Istituzione che fra il III ed il IV secc. perde progressivamente le forme "dell'affettata modestia" escogitata da Ottaviano (Gibbon, 1967, p. 436; Ensslin, 1939, pp. 352-382). Le formule descrittive del principato hanno così lasciato sopravvivere un'illusione: 1'Impero romano, nonostante l'azione disinvolta dell'imperatore, deve continuare ad orientarsi entro il contesto della "libera competizione politica di un'aristocrazia di eguali" (MacCormack, 1995, p. 147; D’Arms, 1990, pp.

\section{Parole chiave}

imperatore ascesa abbigliamento veste imperiale porpora
Keywords

Emperor

Ascension to the Throne Outfit. Imperial Dress Purple 
1. Teja (1993, p. 613); Marotta (1999, p. 57; 2010, pp. 170-188); Hastagnol (1994: 26); Cecconi (2009: 41-91); Cameron (1987, p. 126); Houston (2012). Circa la percezione del linguaggio del vestiario: cfr. Barthes (2006); Flügel (1987); Simmel (2011).
308-320; Millar, 1992). Eppure, le strategie di autorappresentazione virano da un certo punto in poi verso forme assolutistiche, connotate dall'ostentazione di segni che devono illustrare il potere effettivamente detenuto dall'augusto, aprendo al cosiddetto "Dominato".

Perseguendo tale fine si collazionano quelle informazioni utili ad intendere il valore afferito alle insignia nella dinamica della vita dell'Impero e si procede ad una reinterpretazione delle strategie di autorappresentazione imperiale, che orientano un protocollo di ascesa ancora in fieri. Si fa poi luce su quei meccanismi innescati dallostentazione di un preciso abbigliamento e si riconsiderano le implicazioni di un'innovazione fondamentale nel rito d'elezione: la traditio pubblica dei segni del potere.

\section{Le insegne imperiali: la percezione comune ed i valori dell'istitu- zione}

Le strategie di rappresentazione dell'Istituzione imperiale fra i secc. III e IV richiedono un forte rimaneggiamento, supportato dalle più recenti elaborazioni nella dottrina del potere, che si orientano a reimpostare le relazioni fra limperatore, l'aristocrazia ed i sottoposti. La propaganda approntata dalla Tetrarchia ne enfatizza il potere assoluto, senza remora alcuna nel nascondere questa condizione di fatto. ${ }^{1}$ Le ragioni di tale 'cambio di rotta' sono molteplici: l'Impero è appena uscito dalla cosiddetta anarchia militare ed il potere ufficiale ha assunto espressioni sempre meno regolari. Cosa che non è senza conseguenza, ma suscita nei sottoposti incertezza ed una larga sensazione di insicurezza (Reinhold, 1971, pp. 275-302; Jones, 1964). Tale precarietà lascia prevalere una concezione irrazionale del potere e di chi lo detiene, condizione che permea l'immaginario collettivo. Vengono così meno quelle inibizioni che la cultura romana e specialmente senatoriale hanno opposto alla concezione divina del potere sovrano. In questo contesto l'apparizione dell'imperatore assume un impatto più incisivo sui fruitori ed è percepita alla stregua di una vera e propria epifania divina (Eberlein, 1982; Gabelmann, 1984; Alföldi, 1977). Le cerimonie pubbliche richiedono perciò una serie di segni e gesti altamente significanti che devono dichiarare tale natura celeste. Le soluzioni formali con cui il potere costituito si rappresenta ai sottoposti sono oberate di una funzione sostanziale e ciò non deve meravigliare: quelle strategie rispondono ad un'inclinazione propria dei romani, che è stato "il popolo dell'antichità più attento allo status symbol" (Reinhold, 1970, p. 72; Virgilio, 2003; Price, 1984, pp. 5-11; McCormick, 1985, pp. 1-20).

Una necessità che stimola nuove elaborazioni e spinge ad introdurre nell'Impero l'externus et regius mos. Aurelio Vittore riferisce che Diocleziano si appropria del fastoso rito di proskýnesis, tipico del costume persiano, che lo fa assurgere a dio (Aurelio Vittore, Epitome 39.2; Horst, 1932; Babut, 1916, pp. 226-229; Stern, 1954, pp. 184-189). Ammiano stigmatizza questo costume alloctono, che deve risultare piuttosto odioso per lui ed il suo pubblico aristocratico (Ammiano Marcellino, Le storie 15.5.18). Da essi dissente Lattanzio, il quale sostiene che le consuetudini persiane vengono introdotte da Galerio dopo la vittoria del 298 (Lattanzio, Sulla morte dei persecutori 21.2). Una tesi, quella di Lattanzio, che si intende meglio alla luce dellidea che ispira il De morte persecutorum: l'enfatizzazione del mal governo di Galerio. In tal modo si solleva Diocleziano da ogni scelta impopolare che ha connotato il sistema tetrarchico. Nonostante il parere difforme di Lattanzio, si può sostenere che Diocleziano pone in essere una serie di operazioni utili a rafforzare il carattere autocratico dell'Istituzione imperiale, proponendo linnesto di elementi culturali esogeni che sono omogenizzati entro il contesto del cerimoniale pubblico. 
Quest'operazione deve necessariamente investire anche il novero dei segni con cui il potere costituito si mostra ai sottoposti. Finché limperatore si considera il primus inter pares fra i senatori, il suo abbigliamento deve ovviamente omologarsi a quello di coloro con cui condivide la stessa dignità. Tuttavia, il venir meno della "maschera repubblicana" permette l'assunzione di segni che devono distinguerlo dal resto dei membri del Senato (Teja, 1993, pp. 613, 635). E se Eutropio stigmatizza l'abuso dei distintivi di status da parte di Diocleziano (Eutropio, Breviario, 9.26), tale innovazione sembra essere più antica. Aurelio Vittore sostiene che un simile maquillage si deve ad Aureliano (Aurelio Vittore, Epitome 39.2).

Labito indossato dall'imperatore inizia così ad arricchirsi di una serie di dettagli preziosi, quali una sempre maggiore quantità di porpora e la profusione dell’oro. Indossa così la clamide purpurea, un ampio manto di origine militare che nell'Alto Impero viene definito paludamentum. Questo è fermato sulla spalla destra da un grosso spillone in oro decorato di pietre preziose: la fibula, quale segno di esclusiva pertinenza imperiale (Giovanni Crisostomo, De perfecta caritate PG 56, 287a). Sotto di essa porta una tunica di seta, la paragunda, ornata da clavi aurei e stretta alla vita dal cingulum, una cinta anche essa color porpora, che è un altro segno di distinzione e vanta pure un'origine militare. L'abbigliamento viene completato dai sandali purpurei ornati anche questi di pietre preziose. La natura ultraterrena della maestà è resa poi percepibile da dettagli ricercati, quali le gemme ed il filato d'oro, che con i loro riflessi creano un'esperienza sensibile della "luce divina" (Avgoloupi, 2014; Borrego y Vega, 2014, pp. 374-398). Questa si vuole emanata da quellruomo, che le più recenti elaborazioni della dottrina del potere vogliono soprannaturale (Teja, 1993, p. 632). L'introduzione dei materiali preziosi è finalizzata a suggestionare i sottoposti presenti alle apparizioni imperiali e deve certificare l'eccellenza acquisita dal rango imperiale rispetto alla gerarchia senatoriale.

Il processo di evidenziazione della figura dell'augusto non può essere completo senza un segno che coinvolge il capo e la sua sacralità, quale più potente fra gli evidenziatori visuali. Secondo l'Epitome sui Cesari è ancora Aureliano che introduce il diadema (Aurelio Vittore, Epitome 35.5). Questo ornamento aulico sembra derivare dalla benda bianca che cinge le tempie dei sovrani ellenistici ed assume dalla commemorazione dei Ventennalia di Costantino la forma di un cerchio d'oro coperto di pietre preziose e perle o, persino, di una benda riccamente decorata.

L'azione di Diocleziano, dunque, funge da 'catalizzatore' nella costruzione di un abbigliamento che esalta la maestà dellimperatore ed afferma fortemente l'eccellenza dellistituzione, mentre le soluzioni rappresentative costantiniane portano a compimento un processo di esclusivizzazione dei segni di certificazione del rango.

Le insegne costituiscono dunque un sottoprodotto delliniziazione al 'mistero della regalità' (Bourdieu, 1983, pp. 17-18; Carile, 2002b, pp. 75-96). Verificano eccellenza e virtuosità dell'eletto, dimostrando sotto la Tetrarchia il possesso da parte dell'imperatore della Themistes (il diritto divino di nascita). Col cristianesimo poi fungono da criterio utile alla ricognizione della grazia e del consenso divino che conducono al trono (Summer Maine, 1998, p. 7).

Eppure, la selezione di opportuni materiali, fogge e colori costituisce un espediente fallimentare, perché non convince l'aristocrazia. Non si può certo pensare che i senatori o gli intellettuali credano davvero alla divinità dellimperatore. Costoro la considerano piuttosto una "favola" per il volgo, sebbene in pubblico vi aderiscano senza particolari remore; costoro s'afferiscono piuttosto alla dottrina della "doppia verità" teorizzata dal neoplatonismo ed accettata pure dal cristianesimo. Uno stratagemma capace di salvaguardare la dignità dell'Istituzione e pure la libertà di pensiero di nobili ed eruditi (Sinesio, Epistole, 80-95). 
2. Marotta (2010, pp. 170-188; 1999, p. 57; 2016); Kolb (2001, pp.

38-41); MacCormack (1972, pp. 721-752); Tantillo (2015, pp. 543-584).

\section{Costantino e la porpora: l'“abbozzo di un rito" pubblico di ascesa}

Le insegne del potere costituiscono dei lemmi fondamentali nella costruzione del discorso tra imperatore e sottoposti, poiché sono espressione di quel corredo simbolico che rende i sovrani quel che sono ed attinge ai "valori della stirpe, di ammonimento, di forza militare e magica" (Geraci y Marcone, 2004, p. 37). Nondimeno, solo dallelezione di Costantino viene immaginato un rito pubblico di traditio delle insegne dal predecessore al successore. ${ }^{2}$ Tale gestualità, con buona probabilità viene inserita nel rito di ascesa, poiché si comprende che in un periodo di generale insicurezza ed instabilità, la presa di possesso dei segni già ostentati da un imperatore appare capace di rassicurare i sottoposti, costituendo una sorta di garanzia di continuità sul piano materiale. In tal modo si viene ulteriormente incontro ai bisogni profondi della collettività, offrendo messaggi confortanti.

La gestualità della traditio dellinsegna primaria del potere, il manto di porpora, irrompe nella trama di un protocollo che tra il III ed il IV secc. è ancora allo stato embrionale, costituendo parte dell'“abbozzo di un rito" (MacCormack, 1995, pp. 276-277). Le circostanze in cui si realizzano le elezioni del Basso Impero non permettono la consolidazione di un cerimoniale, ma il protocollo risponde alle circostanze in cui matura lelezione. $\mathrm{Al}$ Senato di Roma si affida un ruolo legittimante, poiché convalida la scelta delle truppe, che sempre più spesso si realizza nelle lontane provincie, laddove le legioni innalzano al locus Augusti il proprio comandante, salutandolo con l'adclamatio, che assume un carattere 'quasi' costituzionale (Kelly, 1998, pp. 138-183, 2004; Heather, 1998, p. 189; Paterson, 2007, pp. 121-156). Il sistema tetrarchico cerca di porre rimedio a questa situazione, prevedendo un'elezione per cooptazione da parte del Massimo Augusto del candidato ritenuto meritevole, sicché il consenso assume funzione costituzionalmente vincolante. Tuttavia, tale sistema va presto in crisi e persino i panegirici lasciano trasparire un certo disagio, quando devono giustificare le forzature dei meccanismi costituzionali. Queste controverse situazioni lasciano emergere una formula retorica destinata al successo: l'elezione per volontà divina. Una soluzione che si adatta bene al background religioso, sia esso gentile o cristiano, poiché le elaborazioni del periodo accettano di buon grado che la scelta dei soldati venga ispirata da una qualsivoglia divinità. Questrispirazione è ancor più utile, poiché allontana lo spettro dell'ambizione dall'eletto.

Più specificatamente, fino all'ascesa di Costantino le insegne vengono prodotte per ogni singola elezione e non esiste un cerimoniale pubblico che prevede il passaggio dei simboli del potere, atto a convalidare leelezione. La morte di Costanzo Cloro vede il definitivo disfacimento del sistema tetrarchico, poiché all'assunzione del locus Augusti da parte di Costantino si contrappone la pretesa di Massenzio, figlio di Massimiano, che si installa a Roma. Entro le rivendicazioni dei figli che tentano di succedere ai padri invocando il diritto di nascita, avverso al criterio della libera elezione, si inserisce l'azione dei panegiristi e del vescovo Eusebio, i quali propongono soluzioni retoriche utili a sottolineare la legittimità della pretesa.

Quel vescovo nella Vita Constantini si obera di giustificarne l'ascesa e per far ciò sorvola del tutto la forzatura operata sul meccanismo di cooptazione tetrarchico. Quale attento conoscitore delle prassi costituzionali romane sa bene che la legittimità dell'elezione è garantita solo dal consenso del Massimo Augusto. Per questo descrive lincontro tra limperatore-padre ed il figlio Costantino in cui matura la cooptazione. Una presunta nomina proferita però in un contesto del tutto privato, laddove un padre, per di più dal letto di morte, cerca di favoreggiare il proprio discendente. Una scena struggente di amore filiale e pietà genitoriale, che deve necessariamente suscitare l'empatia nel pubblico. Eusebio sfrutta l'elemento patetico e preferisce descrivere l'intimità di una famiglia e non un atto pubblico e solenne con funzione convalidante, che non si è mai realizzato (Eusebio di Cesarea, Vita di Costantino 1.21.2). 
I buoni sentimenti di un padre, tuttavia, non bastano a fare del figlio un imperatore legittimo. Eusebio perciò deve aggiungere un dettaglio pieno di implicazioni: la consegna della porpora paterna, che negli effetti viene imposta a Costantino prima della presentazione innanzi le truppe. Un atto che deve ratificare formalmente e visivamente la pretesa d'accesso alla carica, riempiendo quel vuoto lasciato dall'assenza di una cerimonia pubblica di cooptazione. Un gesto altamente significante che rafforza la posizione di Costantino e deve allontanare ogni sospetto sulla correttezza del suo agire. Eppure tale dettaglio dimostra quanto il governo dell'Impero sia oramai liquidabile come un vero e proprio 'affare di famiglia' (Rhoads, 2016; Dvornik, 1966).

La descrizione di Eusebio ha dunque il merito di 'fotografare' il momento in cui le insegne acquisiscono una rilevanza propria nel rito di ascesa, mentre prima fungevano da semplice corollario e la loro assunzione era un fatto formale, meramente esteriore potremmo dire, senza troppe implicazioni nella cerimonia pubblica. Il possesso dei segni del potere appartenuti al predecessore sembra assumere da questo momento in poi un carattere legittimante. Tale strategia di comunicazione costituisce un potente indicatore del valore effettivamente percepito da parte dei lettori. ${ }^{3}$

Eusebio, non a caso, sottolinea in modo martellante la proprietà dellinsegna in ogni fase del rito improvvisato. Ha cura di precisare che si consegna a Costantino la "porpora paterna", non un'altra e proprio questa è oggetto di vestizione. L'eletto poi si mostra innanzi alle truppe con linsegna genitoriale, mentre sosta dinnanzi al palazzo avito (Eusebio di Cesarea, Vita di Costantino 1.22.1). Si può postulare che la traditio del segno primario del potere tra padre e figlio possa costituire il primo atto di affermazione del criterio dinastico, che progressivamente si impone sulla libera elezione. Una giustificazione ideologica che si aggiunge alla mera opportunità, poiché quel manto è immediatamente disponibile.

Per l'ascesa di Costantino vengono ad essere "cuciti" in un unico cerimoniale, seppur estemporaneo, i riti connessi all'espulsione dalla società del predecessore e quelli concernenti l'ascesa del successore; il protocollo approntato rivendica così la legittimità della carica assunta. Quest" "abbozzo" di rito viene progettato tenendo conto delle aspettative dei fruitori: popolo e truppe. Una serie di gesti che Eusebio può raccontare circa trent’anni dopo, rielaborandoli attraverso un'operazione puntuale sulla memoria. Un'azione che non esclude qualche tentativo di revisione dei fatti, che viene incontro alla coscienza simbolica del pubblico. Cosa che non stupisce. Tutta la Vita Constantini esprime il laborioso intervento di quel vescovo, che tenta di interpretare il vissuto imperiale per presentarlo nel migliore dei modi possibili. Un'attività che comprende anche i grandi silenzi su alcuni accadimenti e persino lespunzione di fatti difficili da presentare in una luce positiva (Barnes, 1981; Bardill, 2012).

Eusebio sceglie poi di enfatizzare la somiglianza fisica di Costantino col genitore e predica una vera e propria homoiōma, che dimostra la filiazione. Ciò gli permette di sostenere che l'eletto, rivestito delle insegne paterne, possa apparire ai soldati partecipanti alla cerimonia come il defunto genitore appena risorto dall'Ade (Eusebio di Cesarea, Vita di Costantino 1.22.1). Lespediente retorico non si limita a rifunzionalizzare il mito ellenico dell'eterna Basileia, ma ne dimostra pure la filiazione naturale. Tale formula deve rimediare ad un'ulteriore problematica: i controversi natali di Costantino. Eutropio difatti qualifica "oscuro matrimonio" (Eutropio, Breviaro 10.2; Marcone, 2002, p. 20) quello da cui questi viene generato. L'homoioma col genitore deve acquietare tutte le illazioni, che lo delegittimano in ragione del concubinato in cui ha versato la madre.

Per avvalorare ulteriormente la liceità dell'ascesa, Eusebio si preoccupa di introdurre un criterio estraneo alla consuetudine romana: listituto ebraico della primogenitura.
3. MacCormack (1995, pp. 271-273); Calderone (1973, pp. 215-261); Grabar (1936); Treitinger (1956); Pertusi (1991); Carile (2002b, pp. 75-96; 2002c, pp. 53-95; 2003, pp. 589-656); Dagron (1995). Per le insegne si veda: Pertusi (1976, pp. 481-568); Odorico (2005, pp. 1013-1057); Di Cosmo (2009, 2018); Tantillo (2011); Ricci (2011, pp. 191198). Per la porpora: Alberti (2006, pp. 733-736); Bell et alii (1962, p. 35, r. 8-9); Bessone (1996, pp. 142-202); Avery (1940, pp. 66-80); Carile (2000, pp. 65-124; 2002a, pp. 243-269); Wueste (2017); Schramm (1956, pp. 135-156); Chneider (1995, coll. 2000-2020); Dagron (1994, pp. 105-142); Schramm (1956, pp. 135-156); Scott (2009); Steigerwald (1990, pp. 209-239). 
Ricorre pure ad un altro costume biblico posto a suo corollario: la benedizione paterna (Eusebio di Cesarea, Vita di Costantino 1.21.2). Questo perché il vescovo si mostra cosciente della forzatura operata. Per legittimare ulteriormente la posizione di Costantino propone un'espressione rituale che costituisce una sorta di contraltare alla cooptazione da parte del Maximus Augustus, ma anche un espediente utile a rafforzare il valore ricognitivo dell'atto costituzionalmente vincolante d'elezione. La citazione del consenso e della benedizione ha lo scopo di soddisfare sia il lettore avvezzo ai meccanismi del diritto romano, sia il cristiano sensibile alle evocazioni bibliche. A dire del vescovo, quest'imperatore romano decide di ossequiare il diritto di primogenitura previsto dalla legge ebraica, riconoscendolo conforme alla "legge di natura". La precisazione viene inserita per convincere il lettore più avvezzo alle Sacre Scritture ed ha carattere provvidenziale, tanto da evocare la chiamata divina all.Impero. Riferisce poi che il potere imperiale viene trasmesso direttamente dal Massimo Augusto attraverso il consenso prestato sul letto di morte. Si soddisfano così i criteri costituzionali, affinché anche il più esperto di diritto non abbia nulla da obbiettare. Il consenso, nondimeno, viene rafforzato dalla concessione della benedizione paterna. Il precedente biblico, posto a contrappunto delle procedure costituzionalmente vincolanti, lascia percepire che quella prassi non sia ritenuta in grado di persuadere i giurisperiti. Si raffronta un'ansia di legittimità a cui si oppone l'immaginazione del biografo, che riempie con l'estro quei vuoti concernenti una procedura meramente militare, su cui molti anni dopo 'pesano' ancora forti dubbi di costituzionalità.

Questo resoconto ex post, altamente meditato da Eusebio e prodotto per la generazione successiva all'accadimento dei fatti, conserva una certa attendibilità storica, perché collima in più punti con la cerimonia descritta nel panegirico per l'ascesa del 310. Il componimento afferma che, all'acclamazione dei soldati col suo valore 'quasi' costituzionale, si aggiunge la consegna dei simboli del potere paterno da parte delle truppe entro il contesto di una cerimonia pubblica improvvisata. Segue l'apparizione dell'eletto presso le porte del palazzo imperiale e lingresso nello stesso, quale atto di presa di possesso della sede (Panegirici Latini 7.8.2; 7.2.3; 7.4.1). Anche il panegirico del 310 insiste sullidea della filiazione, come ulteriore elemento legittimante (Panegirici Latini 6.3.4; 7.4.2). Eusebio dunque non inventa nulla, ma rielabora il materiale apologetico prodotto nellimminenza dei fatti. Il retore sostiene poi che il dies natalis imperii di Costantino non si concretizza nel giorno dell'elezione, ma può essere addirittura anticipato alla sua nascita, in quanto questi è titolare della cosiddetta fortuna imperii (Panegirici Latini 7.2.2; 7.3.1). Il suo accesso al regno viene persino ricondotto ad espressione di "un'antica prerogativa difamiglia" (Panegirici Latini 7.2.4), che tradizionalmente si fa risalire a Claudio gotico. Un elemento non certo secondario, che lo contraddistingue da tutti i colleghi. Anzi il criterio del sangue lo eleva sopra di loro. Eppure, siamo di fronte ad un espediente della tradizione, che rifunzionalizza la Themistes tetrarchica, quale vera e propria 'vocazione'.

L'elezione viene immaginata alla stregua di un'autonoma azione delle truppe, "senza intrighi, népropaganda elettorale" e presentata come del tutto indipendente da "un consenso fortuito degli uomini" e da "un'improvvisa esplosione di popolarità" (Panegirici Latini 7.3.1; 7.2.5). Per rendere più credibile l'assunto si introduce un potente contrappeso: il pianto dell'eletto, quale segno di abnegazione ed estraneità ai fatti. Un espediente che non può avere molto successo innanzi ad un pubblico romano, che lo trova certamente deleterio, nonostante trasmetta un'immagine efficace, capace di evocare l'estemporaneità dei sentimenti. Per questo segue il bonario invito a non spendere lacrime, perché non adeguate ad un momento così felice (Panegirici Latini 7.8.2).

L'elezione all'Impero viene rappresentata come frutto della scelta ad nutum degli dei, mentre la nomina non è conseguenza della sua bramosia di potere, ma questi ottiene 
l'Impero giacché vi è destinato. L'espressione "universi omnes" adoperata nel panegirico comprova l'effettività della divina ispirazione che muove le truppe.

Il panegirico si preoccupa persino di giustificare l'ascesa sul piano religioso e per questo desina nel raccontare la divinizzazione di Costanzo, che ascende al cielo. Questi, dopo la dextrarum iunctio con Giove, nomina davanti agli dei il figlio Costantino quale Augusto (Panegirici Latini 7.7.3).

Quella del vescovo può essere così qualificata come una versione compatibile col pensiero cristiano dell'ascesa costantiniana, poiché utilizza elementi del contesto biblico per aggiornare le soluzioni retoriche approntate nel 310, che rimandano ad un immaginario tutto gentile.

Entrambi i racconti concordano su un dato di fatto: limprovvisazione di un rito che prevede la consegna dellinsegna primaria del potere. Dimostrano poi che il corredo utilizzato per identificare l'augusto ha assunto un valore fondamentale nelle strategie di autorappresentazione. La traditio della porpora, non solo sembra fornire pregevolezza alla teorizzazione di un diritto alla successione per nascita, ma appare piuttosto efficace sul piano visivo e si radica presso il pubblico.

\section{L'“avita purpura": una prerogativa di famiglia con funzione legittimante}

Eusebio come già accennato ha dovuto affrontare il problema della successione susseguente la dipartita di Costantino. Questrultimo sin dallinizio impronta il suo governo ad un forte carattere dinastico e quel vescovo ha ben presente il marco famigliare con cui questi gestisce la Respublica. Allorché narra la graduale cooptazione della di lui prole, non si pone alcun problema nell'affermare che Costantino riserva l'Impero quale "eredità (...)-per-i suoi diletti rampolli come un bene difamiglia" (Eusebio di Cesarea, Vita di Costantino 4.51.1). Costantino II viene innalzato al rango di cesare in occasione dei Decennalia, Costanzo invece per i Ventennalia, mentre Constante ottieni il titolo nei Trentennalia del regno (Panegirici latini $2.14 .9 ; 10.3 .4$ ). Eusebio precisa poi come Costantino si sia preoccupato di sottoporre i successori ad una adeguata educazione militare, politica, giuridica ed artistica, a cui non manca di aggiungere le nozioni della fede. Fornisce poi una serie di informazioni che tentano di convincere il suo auditorio circa l'adeguatezza della Gens Flavia a detenere il trono, in quanto stirpe 'eletta' da Dio. Tali dettagli non fanno altro che confermare quanto già rappresentato nella De laudibus Constantini, che propone pure la metafora delleterno "fiorire" del "tronco" di Costantino attraverso l'associazione graduale dei figli (Eusebio di Cesarea, Encomio di Costantino 3.1). Ancor più efficace sul piano dellimmaginario appare levocazione di un Costantino raffigurato come auriga solare, che continua a guidare la sua discendenza, simboleggiata da puledri, di cui ne modera lardore, inducendoli alla concordia (Eusebio di Cesarea, Encomio di Costantino 3.2; Karayannopulos, 1956, pp. 341-357).

Una soluzione retorica che rimanda alle rappresentazioni numismatiche della propaganda. Queste vedono Costantino intronizzato ed in vesti civili fra i figli o armato, in piedi, e collocato con loro davanti all'arco che rappresenta la porta del Palazzo. Un criterio dinastico, che appare chiaro sin da subito ai retori. Il panegirico del 313 poi si conclude con una supplica al summus rerum sator e chiede la benedizione di una discendenza numerosa, che permetta a Costantino di continuare a regnare e di stare alla guida del "timone" del mondo (Panegirici Latini 9.26.4). Si ricorre allora ad un locus che evoca i meccanismi costituzionali e riconosce la volontà in Costantino di istaurare una dinastia. 
4. MacCormack (1995, p. 279). Cfr. Socrate Scolastico, Storia Ecclesiastica 1.28-29.
Nazario, nel panegirico del 321 proclamato per i Quindicennalia di Costantino ed i Quinquennalia di Crispo e Costantino II, afferma che questi in ragione dell'associazione dei figli può assurgere al ruolo di Maximus imperator (Panegirici Latini 9.36.5); un'elaborazione recepita solo dal 329 sulle monete, che mostrano l'iscrizione: CONSTANTINUS MAX AUG (MacCormack, 1995, p. 278).

Eusebio, nonostante abbia vissuto in prima persona i disordini avvenuti alla morte dellimperatore, reinterpreta la realtà ed arriva a sostenere che Costantino II, Costanzo II e Costante vengono eletti augusti al momento della morte del padre da tutte le truppe presenti nell'Impero, quasi spiritu sufflati e per diretta volontà di Dio. Il vescovo per migliorare l'efficacia della narrazione afferma che costoro "risplendono cinti degli ornamenti che furono del padre loro" (Eusebio di Cesarea, Vita di Costantino I.2).

Si ripropone nuovamente il valore legittimante delle insegne paterne, che si affianca al consenso prestato dal Massimo Augusto, quale criterio costituzionalmente vincolante, ed all adclamatio militare afferita per divina ispirazione. Una formula di descrizione che, in quanto ecclesiastico, conosce bene e rimanda alla nomina dei vescovi per acclamazione popolare. Insiste poi sulla concordia fra gli eredi del defunto. ${ }^{4}$ Tanto che il loro governo viene rappresentato addirittura migliore di quello del padre, poiché lincisività della sua azione pare moltiplicarsi. Quest’aulica rappresentazione edulcora l'epilogo di una tragedia famigliare e dimostra tutta la sua abilità nel falsificare la realtà. E se le elaborazioni del periodo rendono davvero difficile negare lispirazione divina nell'elezione dellimperatore e dei suoi colleghi, si comprende come gli storici ed i retori sentano la necessità di utilizzare questa formula per ottimizzare il proprio discorso, fin tanto che si cristallizza in un locus tipico dell'ascesa, a cui corollario si può porre il criterio dinastico.

Eusebio è l'unico che si preoccupa di fissare le coordinate temporali dell'accesso all'Impero dei discendenti di Costantino, gli altri panegiristi invece si limitano ad evocare i vota tenuti nelle commemorazioni ufficiali, quale espediente che rende indefinito il loro dies natalis imperii, ma lo rappresenta piuttosto quale realtà in divenire. Uno stratagemma atto a convincere lauditorio che la felicità di quel singolo giorno viene prolungata per tutto il governo, sin da quando sono nominati cesari e non si esaurisce entro la cornice dei riti di ascesa.

Una soluzione che non può però essere esente da critica. Con piglio ironico Ablabio, prefetto del pretorio ed uomo di fiducia dellimperatore, rivisita l'età di Costantino, affermando: "Saturni aurea secla quis requirat? Sunt haec gemmea, sed Neroniana" (Sidonio Apollinnare, Epistole 8). La parossistica rappresentazione di un'età migliore addirittura di quella aurea, poiché di 'gemme', non è altro che un artificio. La propagandata felicitas serve a nascondere quello che, in fin dei conti, è percepito dalla stessa burocrazia come il governo di un tiranno, che soggioga i sottoposti alla stregua di schiavi. Segno di tale scadimento della libertà è il diretto riferimento allintroduzione di un'insegna preziosa come la tenié, quale barbara esagerazione da persiani. Una limitazione personale che si esprime nelle sanguinarie 'purghe' dei nemici dell'augusto, che rimembrano lo spettro della truculenza dei tempi neroniani. 'Purghe' come quella successiva alla morte di Costantino ed operata dai suoi discendenti che coinvolge lo stesso Ablabio.

La formula dellispirazione divina, sebbene estremamente versatile, non viene recepita dal pagano Libanio, il quale scrive un'orazione per Costanzo II e Costante, gli unici sopravvissuti ai disordini del 337 (Libanio, Orazioni, 59.13). Tale componimento vede lenfatizzazione del criterio dinastico e ricorda come Costantino abbia educato la prole a governare, affiancandola gradualmente a sé. La condivisione del potere tra padre e figli assurge a 'clausola di garanzia' per il corretto e soprattutto legittimo esercizio dellimperium, poiché, sottolinea Libanio, nessuna istituzione ha necessità di avere il 
proprio principio nella totale legalità come accade per l'assunzione del locus Augusti. Anche Giuliano nelle due orazioni dedicate a Costanzo II rinuncia al criterio dellispirazione divina, ma ricorre al principio dinastico per legittimarne la posizione. Il panegirico del 356 celebra la discendenza mitica da Claudio gotico e da Costanzo I, elevato all-Impero direttamente dal Massimo Augusto Diocleziano. Ricorda poi come il cugino sia stato educato dal padre Costantino per succedergli al comando. Riferisce pure che è la mitezza di Costanzo a limitare la sua pronta reazione verso i soprusi causati dai fratelli dopo il 337 (Giuliano Imperatore, Orazioni 1.5-7; Kojève, 1998; Tantillo, 1997). Il componimento del 358 evoca ancora il principio dinastico attraverso la proposizione di una metafora omerica: quella dello scettro di Agamennone, una reliquia acheropita del potere, che Giove consegna a Pelope e viene trasmessa nella famiglia per tre generazioni (Giuliano Imperatore, Orazioni, 3.2). Così accade anche al locus Augusti, che viene condiviso da tre generazioni della famiglia di Costantino. Il topos omerico dimostra l'effettiva percezione del valore legittimante delle insegne da parte dell'auditorio, che ormai ha metabolizzato la gestualità della trasmissione dei segni del potere. Se ne deduce che la menzione del principio dinastico e l'evocazione della trasmissione della porpora detenuta dai membri della famiglia costituiscano il contraltare l'uno dell'altra.

Il topos del consenso divino suggestiona anche gli scrittori pagani, tanto da convincerli ad asserire che l'ascesa di Giuliano è voluta dagli dei. Tale soluzione la ritroviamo nel più tardo Ammiano che, raccontando la promozione a cesare di Giuliano avvenuta a Milano nel novembre del 335, ricorre a questo locus. Per la sua nomina Costanzo II organizza una cerimonia prettamente militare volta allaccaparramento del consenso delle truppe verso l'unico sopravvissuto della famiglia allargata di Costantino. La cerimonia si struttura nella seguente sequenza: convocazione e schieramento delle legioni, presentazione dell'eletto da parte del Massimo Augusto per giustificare la propria scelta, vestizione ed adclamatio delle truppe. Il rito si conclude con l'accesso nella carrozza imperiale e l'entrata nel Palazzo, quale presa di possesso. Entro questo schema Ammiano reintroduce la vocazione divina all'Impero, allorché i soldati divinamente ispirati interrompono il discorso dell'augusto, affermando che "la decisione non era frutto di un ridea umana ma ordine della somma divinità". Il racconto viene ulteriormente colorato da un diretto riferimento al principio dinastico, allorché si menziona limposizione dell" "avita purpura", il manto di porpora rivestito dagli antenati, che viene caricato di un'ulteriore funzione legittimante. Funzionalità che si somma alla corretta esecuzione della procedura costituzionalmente vincolante (Ammiano, Le storie 15.8.9).

Il consenso umano diviene allora un indicatore della volontà divina, ma anche una sua immediata esplicazione. Un'elaborazione, quella del cosiddetto consensus omnium, che la tradizione romana adopera come rafforzativo della posizione delleletto. Questo configura un ulteriore corollario, inserito in un pacchetto di soluzioni consuete ed utili a giustificare l'ascesa entro un contesto di legittimità.

Questa versione dei fatti costituisce il contraltare delle narrazioni vescovili dell'ascesa dei sovrani cristiani e può essere intesa come un tentativo di sistematizzazione expost degli accadimenti; una soluzione efficace che è capace di espungere l'intervento del Dio cristiano, lasciando spazio a quelli pagani. Ammiano precisa un ulteriore dato: il matrimonio con Elena, sorella di Costanzo, che deve ribadire il concetto dinastico, rendendolo ben più stringente. Ancora una volta la gestione dell.Impero si risolve in un 'affare di famiglia', mentre il matrimonio incorpora stricto sensu Giuliano nella discendenza di Costantino, quel lignaggio che ha eliminato fisicamente il ramo cadetto della Gens Flavia a cui lui stesso appartiene.

Il ricorso al criterio dinastico deve comunque costituire un espediente di successo, che viene ben compreso dalla nobiltas, che punta all'accaparramento delle magistrature, 
5. Giuliano imperatore, Lettera agli Ateniesi 284b-285a; Labriola (1991, pp. 179-204); Scrofani (2010); Caltabiano (1991); Gnoli (2015); Baynes (1937, pp. 22-29). quale strumento di espansione e conservazione delle prerogative di famiglia. In quest'ottica si spiega l'efficacia della gestualità connessa alla traditio dei segni del potere appartenuti alla gens, poiché l'espediente retorico soddisfa la percezione di quell'aristocrazia destinataria privilegiata di questi componimenti, che suole conservare gelosamente i privilegi famigliari.

\section{Giuliano e le innovazioni nei riti di ascesa: insegne e cerimoniale castrense}

Una più precisa percezione del valore delle insegne sembra emergere durante il cerimoniale improvvisato per l'elezione ad augusto di Giuliano. Linsieme dei gesti colora il rito in uno col significato attribuito dai partecipanti alla porpora ed in particolare ad un accessorio come il diadema. Questo, fino ad allora, ha avuto un ruolo marginale nelle strategie di autorappresentazione dell'augusto e solo da quel momento è ritenuto adeguato ad identificare nell'immediato limperatore. Proprio l'utilizzo delle due insegne, seppur entro una serie di gestualità estemporanee e forse non troppo meditate, costituisce la discriminante fra un mero atto d'usurpazione e la messa in opera di un cerimoniale capace di legittimarne la nomina. Sin dai Ventennalia di Costantino questo ornamento viene usato come evidenziatore visuale, ma non assume un preciso significato rituale. Tuttavia, sin da quel rito assurge a simbolo della vocazione divina all-Impero e, pertanto, la sua imposizione inizia ad essere percepita come necessaria dai partecipanti ed è così inserita. Si raffronta un'assoluta novità rispetto alla tradizione ed alliconografia ufficiale, che già contempla la metaforica incoronazione dellimperatore, quale locus atto ad evocare la vittoria; immagine che è spiegata dalla dedica che lo accompagna: VICTORIA AUGG (McCormick,1986).

Il cerimoniale eseguito per l'elezione di Giuliano si compone di gesti ritenuti particolarmente incisivi da coloro che li operano, tanto da costituire una sequenza realmente capace di suggestionare i fruitori e di offrire un precedente da ripetersi nel lungo periodo. Una ragione della sua fortuna può essere individuata nell'estemporaneità dei comportamenti, che stando ad Ammiano ed allo stesso Giuliano sembrano proposti dal basso e, pertanto, rispondono al senso comune. Gesti immediati, semplici e condivisi dalla truppa, che non si oppone, ma prontamente li avvalla con l’unico gesto che è propria prerogativa: l'adclamatio.

L'estemporaneità del comportamento viene stigmatizzata dallo stesso Giuliano, che nella Lettera agli ateniesi racconta i fatti ed offre una propria giustificazione alla cerimonia improvvisata. Anche questi reintroduce la vocazione divina all'Impero e si rappresenta come una mera pedina di un disegno più grande, operato materialmente dai soldati e progettato dalla divinità. ${ }^{5} \mathrm{Ha}$ cura di raccontare tutto il travaglio interiore che lo attraversa nei momenti antecedenti quell'ascesa, al fine di dimostrare come l'elezione non è stata da lui macchinata. La nomina viene accettata perché questi è vinto dalle pressioni degli uomini divinamente ispirati che lo acclamano, ma anche dalle pulsioni suscitate dalle divinità in lui. La sua elezione viene così rappresentata come l'arrendersi di un uomo mite agli dei. Lelemento sovrannaturale, orbene, va valutato entro la mentalità propria della cronologia di riferimento, ma può essere meglio compreso se qualificato come intima suggestione capace di smuoverlo. $\mathrm{Si}$ raffronta un espediente non certo nuovo ed un contraltare dellinstinctu divinitatis che sembra guidare Costantino per tutta la vita. Una soluzione retorica indispensabile a giustificare la forzatura dei meccanismi costituzionali operata da una designazione, che si liquida come mero arbitrio delle truppe. Eppure, Giuliano è cosciente che sta violando i diritti di Costanzo II. Il criterio dellelezione divina appare quale miglior giustificazione per un atto che costituisce una chiara usurpazione e deve essere, 
pertanto, reinterpretato opportunamente. L'espediente apologetico, seppur rientra nella tradizione, pone una serie di problemi non secondari avverso un più generico pubblico, perché improponibile ai cristiani ed ancor più alla gerarchia della Chiesa. Può solo compiacere qualche senatore attaccato al culto tradizionale, che comunque è sempre in grado di distinguere agevolmente la soluzione retorica adoperata da Giuliano.

Anche Ammiano nel raccontare quellelezione non si discosta dal racconto di Giuliano e non ha troppi problemi nel riportare il locus dell ispirazione divina a riprova dell'imprescindibile suggestione apportata dalle soluzioni descrittive poste in essere per i predecessori. Riferisce il racconto di Giuliano, fatto agli amici più intimi, circa la visione del Genius Publicus, che lo ha esortato ad assumere l'Impero, minacciandolo di abbandonarlo in caso di un suo ulteriore rifiuto (Ammiano, Le storie 20.5.10). Il narratore però attribuisce un diverso significato a talune circostanze. Sottolinea il ruolo dei soldati, che fanno pressione su Giuliano, affinché accetti il titolo di Augusto. Tuttavia sostituisce all'ispirazione divina una meno prosastica brama di denaro, poiché costoro anelano alla concessione del donativo usuale. Precisa poi che al momento non si ha disponibilità di un diadema adeguato al capo di Giuliano, vi è solo quello della moglie, troppo stretto e pertanto inadatto. Si ripiega così per il maniakis posto al collo dei soldati, che uno di questi offre per la cerimonia. Un milite, che viene qualificato come dragonarius, lo pone sul capo di Giuliano (Ensslin, 1942, pp. 268-298). La menzione di un anonimo soldato non va liquidata come una leggerezza del narratore, ma può nascondere un abile trucco dello storico, che sommessamente rafforza lidea di un'elezione avvenuta per effettiva volontà divina. Il fatto che nessuno sapesse chi fosse costui sembra suggerire il ruolo di un deus ex machina, il quale funge da 'catalizzatore' nell'evolversi degli eventi. La suggestione di un probabile intervento divino sembra giustificare un gesto del tutto nuovo entro la trama del protocollo di elezione. Un gesto estemporaneo, che a causa della poca autorità di chi lo compie è solitamente destinato alloblio.

Solo pochi mesi dopo i fatti Libanio torna sul valore di quellimprovvisato cerimoniale castrense e interpreta il suo corredo simbolico. Questi però si rivolge alla corte, cosa che gli permette di liquidare l'imposizione del maniakis alla stregua di un ornamento militare e non afferisce alcun valore legittimante all'assunzione del diadema. Il rito viene così ricondotto alla consueta gestualità che segue la commemorazione di una vittoria, sicché può affermare: "beati quei militi (...) che non hanno potuto fare a meno di posare sul tuo capo la corona di pietre preziose" (Libanio, Orazioni 12.59). Ciò dimostra che l'élite non riesce a comprendere affondo la portata del comportamento dei soldati, perciò il panegirista si limita ad evocare sommessamente al suo pubblico delle immagini note, come quelle degli augusti coronati dalle vittorie. Soluzione che non meraviglia, poiché per autorappresentarsi Giuliano sceglie proprio quel locus di vittoria, che inscrive ad esempio sul milarense di Antiochia accompagnato dalla dedica: VICTORIA ROMANORVM (Diegi, 2005; López Sánchez, 2012, pp. 158-182; Varner, 2012, pp. 183-221). Il tipo iconografico fonde il topos del Palazzo, rappresentato da un arco che suole evocarne la porta, con una variante della majestas imperiale, raffigurata frontalmente allo spettatore, mentre declina il capo verso destra. Allimperatore si appropinqua una Vittoria alata, portatrice di corona, che si appresta a conferirgli il diadema, segno della sua felicitas e garanzia del suo buon governo.

Tale limite cognitivo, non può ostacolare l'aggregarsi di nuovi significanti attorno ad un segno come il collare, realizzato per di più in materiale vile, che in origine non ha alcun significato connesso allascesa. ${ }^{6}$ Sorprende poi come un materiale non pregiato e di conseguenza non adeguato alla gerarchia possa acquisire valenza simbolica. Tuttavia il suo senso si spiega solo entro l'alea di un rito castrense ed avverso un
6. Ammiano, Le storie 20.4.17. Questo accessorio, noto pure come torques, è un ornamento etnico indossato non solo dai soldati barbari, ma anche dalle divinità germaniche; cosa che lo rende un segno sacro. L'alea di significatività può spiegare la sua adeguatezza a fungere da sostituto del diadema aureo. Cfr. Ensslin (1942, pp. 268-298). 
novero di fruitori caratterizzato da militi. Un espediente di successo che sopravvivrà senza soluzione di continuità per circa due secoli.

L'imposizione si unisce ad un altro gesto estraneo al cerimoniale romano come linnalzamento sullo scudo, che rimanda allimmaginario del potere dei popoli collocati alla periferia dell'Impero, i quali compongono le truppe affidate al comando di Giuliano in Gallia. Costoro ossequiando le proprie tradizioni pongono in essere il rito con cui si è soliti eleggere i condottieri delle tribù barbare. Non meraviglia che l'elevazione configuri un espediente di successo presso quelle truppe reclutate in Gallia o nell'area del Reno, tanto da costituire un precedente che si ripete per l'usurpatore Firmo (Ammiano Marcellino, Le storie 29.5.20) ed un altro pretendente sostenuto dalla nobiltà gallica. Si evidenzia uno scarto nelle forme di rappresentazione dell'ascesa e l'imporsi di un linguaggio lontano dalla coscienza simbolica del 'centro' dell'Impero. Emerge così un idioma comunicativo sempre più connotato dai particolarismi etnici e dalle esigenze della periferia. Una prassi alloctona che spiega la lunga resistenza di Giuliano, il quale deve allontanare da sé il sospetto di ogni ambizione imperiale. Resistenze che sembrano pure giustificate nell'ottica del pensiero magico, poiché si crede che una proclamazione "notturna" sia considerata nefasta.

Non meraviglia che Ammiano, il quale si rivolge ad un pubblico abituato ai costumi romani, abbia a precisare che il giorno dopo vengano riunite nuovamente le truppe per l'allocuzione dal tribunal, a cui segue la rituale adclamatio, dal valore 'quasi' costituzionale.

Anche Giuliano è ben conscio che l'atto celebrato dall'esercito è privo di valenza costituzionale ed è ritenuto valido solo dalle truppe che l'hanno operato, le quali arrivano a chiedere persino la nomina dei funzionari. Questi, pertanto, non osa mostrarsi in pubblico ornato delle insegne, ma si limita a rivolgersi al cugino Costanzo, Maximus Augustus, e chiede la ratifica di quella situazione di fatto. Invia una lettera accompagnata da una petizione dellesercito volta al riconoscimento della carica afferitagli. Il ricorso alla petizione costituisce un corollario dell'operato di Giuliano, che si orienta al 'politicamente corretto' e vuole meramente dimostrare la propria buona fede.

Nondimeno, nell'imminenza dei fatti l'elezione divina non viene menzionata. Il panegirista Mamertino nel 361 per meglio giustificare la designazione di Giuliano ad imperatore innanzi al Senato di Costantinopoli ricorre piuttosto al principio della virtù, liquidando sia la vocazione divina, che il criterio dinastico (Panegirici Latini 2.27.10; De Vita, 2011). Questi rappresenta la nomina come fatto necessario, poiché Giuliano è il migliore. La meritocrazia sembra così accantonare la parentela con Costantino. Una presa di posizione che rispecchia il pensiero di Giuliano, il quale rifiuta di afferire l'ascesa alla sua famiglia allargata. Una soluzione che offre un buon margine di operabilità sul piano retorico, allorché cerca di recuperare uno dei criteri tetrarchici per la scelta del nuovo imperatore ed apre allopera di interpretazione dei panegiristi.

Un tema, quello della virtù, che torna con Libanio nel panegirico del 362 e gli permette di ricondurre l'elezione di Giuliano ad un atto dovuto, poiché i soldati non hanno fatto altro che consegnare l'Impero a quell'uomo dotato di aretai (Libanio, Orazioni 12.49).

Eppure, tale soluzione estemporanea non deve soddisfare il pubblico. Si osserva come lo stesso Libanio, solo un anno dopo e col panegirico per il consolato del 363 d.C., deve ricorrere al locus dellispirazione divina ed al ruolo della divinità che muove $\mathrm{i}$ soldati e li spinge all'elezione, "poiché il dare e ricevere limpero dipendevano dal volere degli dei" (Libanio, Orazioni 12.49). Questi rimembra il rito d'ascesa e limposizione delle insegne. Non manca di fare riferimento al dettaglio prezioso che le costituisce, 
quali i frutti del mare, come la porpora estratta dal murice e le perle; materiali non alla disponibilità di tutti, che qualificano il rango imperiale per quello che è. Una precisazione da non sottovalutare, poiché il riferimento approntato rimanda a quelle strategie dell'opulenza che hanno il compito di colonizzare linconscio dell'auditorio, suggestionandolo con immagini adeguate. Si rappresenta unidea condivisa della maestà che la morigeratezza dei costumi di Giuliano progressivamente va ad offuscare.

Orbene, con lelezione di Giuliano si fa largo la percezione comune. I più richiedono un imperatore ornato di abiti fastosi, che fungono da evidenziatori visuali. Un vero e proprio paradosso, se si pensa che Giuliano ha una visione peculiare dell'Istituzione imperiale e tende a sminuire quei segni sfarzosi richiesti dalla collettività. Una strategia di rappresentazione orientata alla sobrietà, che però non sembra convincere il pubblico, specie quello formato dal popolo assuefatto ad un dispendio di risorse capace di dimostrare la prosperità dell'augusto (Giuliano imperatore, Misopogon, 3; 4; 10; 19; PratoMicaella, 1975). Una moderazione eccessiva della maestà, che stranamente non compiace nemmeno quell'aristocrazia che ha già sofferto l'alterigia di Diocleziano. Una visione modesta del potere, che viene dimostrata dalla statua ora al Louvre che rappresenta Giuliano in espressione patetica, privo delle insegne e nelle vesti di filosofo. Il tradimento dell'aspettativa sociale non può che generare uno schock visuale ed indebolire quella barriera posta fra popolo e imperatore, costituita dall'apparato di segni con cui l'augusto si presenta al pubblico. ${ }^{7}$ E se tale idea sembra compiacere i circoli filosofici elitari di cui limperatore fa parte, al contrario il grande pubblico si dimostra poco interessato all'ostentazione delle qualità morali dell'augusto. ${ }^{8}$ Ciò non convince nemmeno il compiacente Ammiano, il quale ricorda che Costanzo II ha sempre salvaguardato la dignità della maestà (Ammiano, Le storie 21.16.1).

\section{Insegne e funzione legittimante: l'evoluzione da Gioviano a Valen- tiniano}

La singolare situazione in cui matura l'elezione di Gioviano, a seguito dell'assassino di Giuliano, costituisce il pretesto per il consolidarsi della rituale traditio delle insegne in un cerimoniale che mantiene uno spiccato carattere militare (Baynes, 1937, pp. 22-29). Questa volta però il protocollo non catalizza laccaparramento del consenso attorno al comandante, ma gli alti ufficiali si limitano a designare uno di loro, Gioviano, che viene presentato alle truppe per la formale adclamatio (Socrate Scolastico, Storia Ecclesiastica 3.26). Segue limposizione della porpora del predecessore, quale evidenziatore visuale del rango che stigmatizza la continuità dellistituzione. Proprio la consegna della porpora rivestita dai costantinidi deve riempire l'assenza di nobiltà del lignaggio di Gioviano. Carenza che viene meglio soddisfatta sul piano materiale dallevocazione del legittimo possesso del segno del potere trasmesso entro una famiglia che i panegirici cantano come divina. E ciò a maggior ragione, perché secondo il Nazianzeno quella domus gode onori quasi simili a quelli degli apostoli (Greg. Naz., Contr. Jul. II, Or. V, 17, PG 35, coll. 685-688).

La presa di possesso dellinsegna costituisce poi un espediente che pone rimedio alla mancata espressione di un previo consenso imperiale e fornisce autorità maggiore all'assemblea elettiva. Limposizione del manto purpureo innanzi agli elettori riuniti diventa così il sigillo materiale della legittimità della procedura approntata. E se si comprende bene come quellinsegna fosse immediatamente reperibile ed impiegata nei riti di ascesa, deve apprezzarsi lo sforzo dei panegiristi che devono interpretare gli eventi e offrirgli spiegazioni di comodo. Una soluzione retorica che dimostra come il cerimoniale di ascesa si organizzi attorno alla consegna al successore dellinsegna del predecessore. Sicché l'atto viene percepito dai fruitori come fornito di una capacità convalidante, a riprova dell'avvenuta colonizzazione dellinconscio.
7. Per tradizione all'augusto è concessa solo la facoltà di esercitare la virtù della modes tia, la "sophrosyne". Pertanto, si preferisce condannare ogni alternativa che sminuisce il fasto della maestà, cfr. Socrate Scolastico, Storia Ecclesiastica 3.1.

8. Nel Misopogon Giuliano si autorappresenta come etairos e rinuncia ai titoli quali dominus e despotēs. Cfr. Giuliano imperatore, Misopogon 3, 4. 
9. Kantorowicz (1963, pp. 117-177); MacCormak (1995, pp. 293-295).
Il breve regno di Gioviano si risolve in una mera anabasi da Ctesifonte ad Antiochia e si conclude con la di lui morte a Dadastana nel febbraio del 364.

Il precedente, sebbene avveratosi in un momento di emergenza, rende più facile lelezione del successore: Valentiniano I, che si realizza nello stesso anno a Nicea per mezzo di un'assemblea di dignitari sia civili, che militari.

I panegirici testimoniano come venga perpetuato il cerimoniale castrense posto in essere per Giuliano e culminante nell adclamatio e nella traditio delle insegne. Dimostrano poi il valore acquisito dalle insegne, tanto che la loro assunzione viene rappresentata come una sorta di palingenesi mistica delleletto, realizzata nel marco del fasto dei materiali preziosi assunti e nello splendore emanato da questi. Non meraviglia che Simmaco possa descrivere il rito di ascesa quale eliofania nel panegirico del 369. La presentazione dell'augusto viene paragonata al sorgere del sole ed all'apparizione di una stella, che sorgono dal mare. Il retore recupera così due loci oramai desueti: quello dell'oriens augusti e del divus sidus, ma comunque ben presenti all'auditorio. Una mimesi solare che si realizza in ragione dell'assunzione della clamide. Questa a mezzo del suo cruor lo assimila all" "alba purpurea" con "la sua luce rossa" o ad una stella che declina col suo chiarore rossastro all'orizzonte (Simmaco, Orazioni 1.7; MacMullen, 1964, pp. 435-455; Matthews, 1989, pp. 231-252). Siamo di fronte ad una citazione erudita della tradizione retorica e della propaganda visuale del sec. III a cui Simmaco ricorre, poiché sa che ha avuto un effettivo successo nella cultura materiale e nello specifico nelle soluzioni descrittive destinate alle emissioni numismatiche. ${ }^{9}$

A questa metafora affianca la metamorfosi di Valentiniano, che rivestito del corredo militare vede tramutarsi gli elementi della propria armatura nelle insegne imperiali. L'elmo si converte in diadema, mentre la lancia prende la forma di uno scettro, la fatica della spada poi vale per lui l'oro dell'opulenza imperiale. Questi viene avvolto dai segni del potere, che quasi lo irrigidiscono, fin tanto da poter essere rappresentato all'auditorio come una statua vivente della divinità. Si introduce un'immagine tutta nuova, quella dellimperatore ingioiellato e rigidamente maestoso, che si appresta a diventare un locus della letteratura di genere. Una soluzione non esente da critiche, che più tardi ispirerà Sinesio di Cirene, il quale la deprecherà, evocando la maledizione omerica della tunica di pietra (Sinesio, Sulla regalità 14-15; Iliade 3.57; Teja, 1993 , pp. 638-639).

Segue poi lammonimento rituale, quale potente contrappeso retorico, che ricorda alleletto quanto il sommo dei poteri non porti alcun vantaggio, se non affanni. Un calmiere alla tracotanza che accompagna il locus Augusti, reso indispensabile dalla tradizione e dal cui ricorso Simmaco non è esentato.

Anche Temisto nel panegirico del 364 ricorre allefficacia visuale delle insegne. Eppure sembra dimenticare il loro valore legittimante, ma anzi ricorda allo stesso Valentiniano come sia il possesso di un'aquila d’oro, sia il tributo degli onori siano un corollario dell'elezione; semplici evidenziatori visuali e mere garanzie dell'opulenza imperiale (Temisto, Orazioni 73c-74a). Li riduce a ovvi orpelli esteriori che devono suffragare la più aggiornata rappresentazione dell'Istituzione imperiale: un governo retto dal dio ed officiato dagli uomini. Si predica una monarchia divina, che declina le elaborazioni di Eusebio di Cesarea entro l'alea di uno spirito laico. Lavvertimento, ritualmente afferito, può essere ricondotto alla struttura di un peri basileias o alla stregua di un 'cortese' ammonimento. Sminuendo il valore dei segni del potere, non si fa altro che calmierare lesaltazione causata dal loro possesso. Al contempo, la loro sommessa negazione non fa altro che riconoscerne limprescindibilità, in quanto segni indispensabili a verificare l'effettività della carica. 
Questo discorso intriso di filosofia neoplatonica evoca la cogenza dellelezione divina e si spiega in una serie di esortazioni, che rimembrano allimperatore la natura soprannaturale della sua nomina, poiché essa è considerata come il giudizio degli dei operato dagli uomini. Il componimento offre le chiavi cognitive adoperate dagli eruditi del sec. IV per leggere la vita politica dell.Impero ed illumina circa i criteri utilizzati per interpretare i suoi episodi significanti. Esprime così un giudizio a posteriori, che è volto ad avvallare un'elaborazione come la vocazione divina all'Impero e l'ispirazione celeste dei soldati, presentandoli però come un 'a priori' rispetto all'ascesa. Ossequiando la tradizione, Temisto si propone di persuadere il suo auditorio e sostiene che l'elezione non è affatto un affare umano, facendo salvo il carisma dell'Istituzione. Questo componimento 'canonizza' infine lirrompere della divinità negli affari umani e nella gestione della Respublica. Se fin dalla Tetrarchia la virtù innata dell'eletto costituisce un fatto privato fino al momento della presa di possesso dell'Impero e se è altrettanto privata la visione del Genius Publicus di Giuliano, le cose ora sono ben diverse: siamo di fronte ad un intervento pubblico della divinità che realizza i propri disegni a tramite delle azioni umane, rendendo le truppe strumenti consapevoli. Temisto propone nel prefato panegirico quegli indizi ritenuti indispensabili per riconoscere agevolmente la chiamata divina al governo e dichiara apertamente che gli operatori del rito li hanno riconosciuti tutti come tali.

\section{Conclusioni}

Le contingenze che spingono Diocleziano ad un maquillage dell'Istituzione imperiale permettono alle insegne, destinate fino ad allora alla funzione di prevalente evidenziatore visuale, di assurgere ad effettivi segnacoli del potere. Con Costantino poi la porpora posseduta dal predecessore assume un ruolo legittimante nei riti d'ascesa, specie se realizzati in un contesto che forza il meccanismo di elezione in vigore. $\mathrm{Di}$ conseguenza si osserva l'esponenziale crescita del loro valore, che è proporzionale alla complessità delle contingenze in cui si verifica l'assunzione del locus Augusti. In questo processo di accrescimento di senso gioca un ruolo importante il consolidamento della formula delleelezione divina, che sin da Diocleziano permette il venire meno di quei freni inibitori opposti dalla cultura romana ad un'elaborazione ritenuta una fatuità orientale.

È il possesso da parte dellimperatore che previamente ha regnato a fornire a questi segni un carattere 'legittimante', nonostante queste non siano ritenute di origine divina $\mathrm{o}$, almeno, non sono additate come tali fino al $\mathrm{X}$ sec. allorché il de administrando imperio accoglie una leggenda concernente una corona consegnata direttamente a Costantino da un angelo e collocata nell'altare di S. Sophia a Costantinopoli.

Eppure, la trasmissione dei segni dei predecessori da sola basta ad evocare l'eterna Basileia e permette alle insegne di acquisire il valore di "emblemi imperituri" (Tantillo, 2011, p. 23). Non a caso una simile suggestione torna in Giuliano, il quale per avvalorare la trasmissione del potere allinterno della propria famiglia allargata ricorre all'autorità di Omero e cita lo scettro di Agamennone, dono di Zeus, trasmesso tra i discendenti di Pelope (Iliade 2.100-108). Un ulteriore indicatore capace di dimostrare lo svilupparsi anche a Roma di un criterio dinastico con funzione legittimante, che per essere percepito come tale abbisogna di elementi materiali quali le insegne, a cui si 'appoggia' per apparire più incisivo. Tuttavia, le elaborazioni teoriche non sono ancora in grado di sostenere un diritto del sangue al trono. Il principio della libera elezione, il locus omerico del re nutrito da Zeus ed educato dalle Muse di successo nei panegirici (Temisto, Orazioni 65.b-c), nonché la tradizione neoplatonica che 
sistematizza quelle idee non hanno permesso il sorgere del diritto di una sola famiglia a "sostenere il carisma del potere" (MacCormack, 1995, p. 293). 


\section{Bibliografia}

" Alberti, M. E. (2006). La prima porpora: primato etnico o comune patrimonio mediterraneo?. In Studi di Protostoria in onore di Renato Peroni (733-736). Firenze: All'Insegna del Giglio.

"Alföldi, A. (1977). Die monarchische Repräsentation im römischen Kaiserreiche. Darmstadt: Wissenschaftliche Buchgesellschaft.

»Avery, W. T. (1940). The 'adoratio purpurae' and the Importance of the Imperial Purple in the Fourth Century of the Christian Era. Memoirs of the American Academy in Rome, $17,66-80$.

"Avgoloupi, E. (2014). Simbologia delle gemme imperiali bizantine nella tradizione simbolica mediterranea delle pietre preziose (secoli I-XV d.C.). Spoleto: CISAM.

» Babut, C. (1916). L'adoration des empereurs et les origines de la persecution de Dioclétien. Revue Historique, 133, 226-229.

" Baynes, N. H. (1937). The Death of Julian the Apostate in a Christian Legend. JRS, 27, 22-29.

» Bardill, J. (2012). Constantine, Divine Emperor of the Christian Golden Age. Cambridge: Cambridge University Press.

" Barnes, T.D. (1981). Constantine and Eusebius. Boston: Harvard University Press.

» Barthes, R. (2006). The Language of Fashion. Oxford: Bloomsbury.

» Bell, H. I. et alii (Eds.). (1962). The Abbinues Archive. Papers of a Roman Officer in the Reign of Constantius II. Oxford: Clarendon.

» Bessone, L. (1996). La porpora a Roma. En La porpora, realtà e immaginario di un colore simbolico (142-202). Venezia: Istituto veneto di Scienze, Lettere ed Arti.

» Borrego, P. y Vega, C. (2014). A New Approach to the Understanding of Historic Textil. In M. Harlow e M. L. Nosch (Eds.), Greek and Roman Textiles and Dress: An Interdisciplinary Anthology (374-398). Oxford-Philadelphia: Oxbow Book.

» Bourdieu, P. (1983). La distinzione. Critica sociale del gusto. Bologna: II Mulino.

"Caltabiano, M. (1991). L'epistolario di Giuliano imperatore: saggio storico. Napoli: D’Auria.

" Cameron, A. (1987). The Construction of Court Ritual: the Byzantine Book of Ceremonies. In D. Cannadine e S.F.R. Price (Eds.), Rituals of Royalty: Power and Ceremonial in Traditional Societies (106-136). Cambridge: Cambridge University Press.

» Carile, R. A. (2000). Le insegne del potere a Bisanzio. In La corona e i simboli del potere (65-124). Rimini-Siena: Il Cerchio.

" Carile, R. A. (2002a). Produzione e usi della porpora nell'Impero bizantino. In R.A. Carile (Ed.), Immagine e realtà nel mondo bizantino (243-269). Bologna: Lo Scarabeo.

" Carile, R. A. (2002b). Regalità sacra ed iniziazione nel mondo bizantino. In A. Panaino (Ed.), Sulla soglia del sacro: esoterismo ed iniziazione nelle grandi religioni e nella tradizione massonica. Atti del Convegno di Studi del GOI (75-96). Milano: Mimesis.

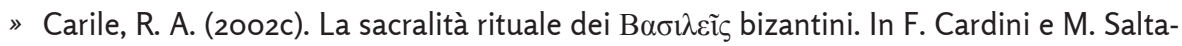
relli (Eds.), Per me reges regnant. La regalità sacra nell'Europa medievale (53-95). Rimini-Siena: Il Cerchio. 
"Carile, R. A. (2003). La prossemica del potere: spazi e distanze nei cerimoniali di corte. In Uomo e spazio nell'Alto Medioevo (589-656). Spoleto: CISAM.

» Hastagnol, A.C. (1994). L'évolution politique du règne de Dioclétien (284-305). Antiquité Tardive, 2, 23-31.

»Cecconi, A. (2009). Da Diocleziano a Costantino: le nuove forme del potere Storia d'Europa e del Mediterraneo. In A. Barbero (Ed.), L'Ecumene romana. L'impero tardoantico (41-91). Roma: Salerno Editore.

» Chneider, K.S. (1995). Purpura. RE, 23, 2, coll. 2000-2020.

"Dagron, G. (1994). Nés dans la pourpre. Travaux et mémoires, 12, 105-142.

»Dagron, G. (1995). Costantinopoli. Nascita di una capitale. Torino: Einaudi.

» D’Arms, J. (1990). The Roman Convivium and the Idea of Equality. In O. Murray (Ed.). Sympotica (308-320). Oxford: Oxford University Press.

»De Vita, M. (2011). Giuliano imperatore filosofo neoplatonico. Milano: Vita e Pensiero.

»Di Cosmo, A. P. (2009). Regalia signa: iconografia e simbologia della potestà Imperiale. Porphyra, International Academic Journal in Byzantine Studies, Extra issue 10.

»Di Cosmo, A.P. (2018). Imperial Iconography of Byzantium. In C. Smith (Ed.), Encyclopedia of Global Archaeology. New York: Springer Press.

»Diegi, R. (2005). Giuliano l'Apostata: una pecora nera nel cristianissimo Impero romano del IV secolo. Monete Antiche, 20, 17-24.

»Dvornik, F. (1966). Early Christian and Byzantine Political Philosophy. Washington D.C.: Dumbarton Oaks.

»Eberlein, J.K. (1982). Apparitio regis, revelatio veritatis. Studien zur Darstellung des Vorhangs in der bildenden Kunst von der Spätantike bis zum Ende des Mittelalters. Wiesbaden: L. Reichert.

» Ensslin, W. (1939). The End of the Principate. In A. Cameron (Ed.), The Cambridge Ancient History, 13 (352-382). Cambridge: Cambridge University Press.

»Ensslin, W. (1942). Zur Torqueskrönung und Schilderhebung bei der Kaiserwahl. Klio, 35, 268-298.

» Flügel, J. C. (1987). Psicologia dell'abbigliamento. Milano: Franco Angeli.

» Gabelmann, H. (1984). Antike Audienz-und Tribunalszenen. Darmstadt: Wissenschaftliche Buchgesellschaft.

» Geraci, G. y Marcone A. (2004). Storia romana. Firenze: Le Monnier.

" Gibbon, E. (1967). Storia della decadenza e caduta dell'impero romano. Torino: Einaudi.

» Gnoli, T. (2015). Le guerre di Giuliano imperatore. Bologna: II Mulino.

» Grabar, A. (1936). L'empereur dans l'art byzantine. Paris: Les Belles Lettres.

» Jones, A.H.M. (1964). The Later Roman Empire, 284-602: a Social, Economic and Administrative Survey. Oxford: Blackwell.

"Heather, P. (1998). Senators and Senates. In A. Cameron (Ed.), The Cambridge Ancient History, 13 (184-210). Cambridge: Cambridge University Press.

» Horst, J. (1932). Proskynein: Zur Anbetung im Urchristentum nach ihrer religionsgeschi-chtlichen Eigenart. Gütersloh: C. Bertelsmann.

» Houston, M. G. (2012). Ancient Greek, Roman and Byzantine Costume and Decoration. London: Courier Corporation. 
Kantorowicz, H. (1963). Oriens Augusti-Lever du roi. Dumbarton Oaks Papers, 17, 117-177.

» Karayannopulos, I. (1956). Konstantin der Große und der Kaiserkult. Historia, 5, 341-357.

» Kelly, C. (1998). Emperors, Government and Bureaucracy. In A. Cameron (Ed.), The Cambridge Ancient History, 13 (138-183). Cambridge: Cambridge University Press.

» Kelly, C. (2004). Ruling the Later Roman Empire. Cambridge: Harvard University Press.

» Kojève, A. (1998). L'imperatore Giuliano e l'arte della scrittura. Roma: Donzelli.

» Kolb, F. (2001). Herrscherideologie in der Spätantike. Berlin: Akademie Verlag.

» Labriola, I. (ed.) (1991). Giuliano Imperatore, Lettera agli ateniesi. InvLuc, 13-14, 179-204.

»López Sánchez, F. (2012). Julian and his Coinage: A very Constantinian Prince. In N. Baker-Brian y S.F. Tougher (Eds.), Emperor and Author: The Writings of Julian 'the Apostate' (159-182). Swansea: The Classical Press of Wales.

» MacCormack, S. G. (1972). Change and Continuity in Late Antiquity. The Ceremony of Adventus. Historia, 21, 721-752.

» MacCormack, S. G. (1995). Arte e cerimoniale nell'antichità. Torino: Einaudi.

» Marotta, V. (1999). Liturgia del potere. Documenti di nomina e cerimonie di investitura fra principato e tardo impero romano. Napoli: Loffredo.

" Marotta, V. (2010). Gli dèi governano il mondo. La trasmissione del potere imperiale in età tetrarchica. Polis. Studi interdisciplinari sul mondo antico, 3, 170-188.

» Marotta, V. (2016). Esercizio e trasmissione del potere imperiale (secoli I-IV d.C.). Torino: Giappichelli.

» Matthews, F. (1989). The Roman Empire of Ammianus. Baltimore: Johns Hopkins University Press.

» McCormick, M. (1985). Analyzing Imperial Ceremonies. Jahrbuch der Österreichischen Gesellschaft für Byzantinistik, 35, 1-20.

» McCormick, M. (1986). Eternal Victory, Triumphal Rulership in late Antiquity, Byzantium and the early Medieval West. Cambridge-Paris: Cambridge University Press.

" MacMullen, R. (1964). Some Pictures in Ammianus Marcellinus. ArtB, 46, 435-155.

» Millar, F. (1992). The Emperor in the Roman World (31 BC-AD 337). Ithaca: Cornell University Press.

» Odorico, P. (2005). Habiller le prince. Vêtements et couleurs à la cour de Byzance. In Comunicare e significare nell'alto Medioevo (1013-1057). Spoleto: CISAM,

» Paterson, J. (2007). Friends in High Places: the Creation of the Court of the Roman Emperor. In A. Pawforth (Ed.), The Court and Court Society in Ancient Monarchies (121-156). Cambridge: Cambridge University Press.

»Pertusi, A. (1976). Insegne del potere sovrano e delegato a Bisanzio e nei paesi di influenza bizantina. In Simboli e simbologia nell'Alto Medioevo (481-568). Spoleto: CISAM.

» Pertusi, A. (1991). Il pensiero politico bizantino. Bologna: Patròn.

» Prato, C. y Micaella, D. (Eds.) (1979). Giuliano Imperatore. Misopogon. Roma: Edizioni d'Ateneo.

»Price, S.R.F. (1984). Rituals and Power. The Roman Imperial Cult in Asia Minor. Cambridge: Cambridge University Press.

» Reinhold, M. (1970). History of Purple as a Status Symbol in Antiquity. Brussels: Latomus. 
" Reinhold, M. (1971). Usurpation of Status and Status Symbols in the Roman Empire. Historia, 20, 275-302.

» Ricci, M. (2011). Elementi per la ricostruzione delle insegne. In C. Panella (Ed.). I segni del potere. Realtà e immaginario della sovranità nella Roma imperiale (191-198). Bari: Edipuglia.

» Rhoads, M. (Ed.). (2016). Imperial Lineages and Legacies in the Eastern Mediterranean: Recording the Imprint of Roman, Byzantine and Ottoman Rule. Birmingham: Taylor \& Francis Group.

» Schramm, P. E. (1956). Herrschaftszeichen und Staatssymbolik. Beiträge zu ihrer Geschichte von dritten bis zum sechzehnten Jh., I-III. Early Medieval Europe, 3, 135-156.

"Scott, M. (2009). Medieval Dress \& Fashion. London: British Library.

"Scrofani, G. (2010). La religione impura: la riforma di Giuliano imperatore. Brescia, Padeia.

» Simmel, G. (2011). Metropoli e moda. Prato: Piano B.

》Summer Maine, H. (1998). Diritto antico. Milano: Giuffrè.

»Smith, R. (2007). The Imperial Court of the Late Roman Empire, c. AD 300-c. AD 450. In A.S. Spawforth (Ed.), The Court and Court Society in Ancient Monarchies (157-232). Cambrige: Cambrige University Press.

"Smith, R. (2011). Measures of Difference: the Fourth-Century Transformation of the Roman Imperial Court. American Journal of Philology, 132, 125-151.

» Steigerwald, G. (1990). Das kaiserliche Purpurprivileg in spätrömischer und frühbyzantinischer Zeit. Jahrbuchfür Antike und Christentum, 33, 209-239.

» Stern H. (1954). Remarkson the Adoratio under Diocletian. Journal of Warburgand Courtland Institute, 27, 184-189.

»Virgilio, B. (2003). Lancia, diadema e porpora. Il re e la regalità ellenistica. Pisa: Giardina.

»Varner, E. R. (2012). Roman Authority, Imperial Authoriality, and Julian's Artistic Program. In N. Baker-Brian e S.F. Tougher (Eds.). Emperor and Author: The Writings of Julian 'the Apostate' (183-211). Swansea: The Classical Press of Wales.

» Tantillo, I. (1997). La prima orazione di Giuliano a Costanzo. Roma: L'Erma di Bretschneider.

» Tantillo, I. (2011). Insegne e legittimazione nell'impero romano. In C. Panella (Ed.), I segni del potere. Realtà e immaginario della sovranità nella Roma imperiale (13-24). Bari: Edipuglia.

» Tantillo, I. (2015). I cerimoniali di corte in età tardoromana (284-395 d.C.). En Le corti nell'alto medioevo (543-584). Spoleto: CISAM.

» Teja, R. (1993). Il cerimoniale imperiale. In A. Carandini et alii (Eds.), Storia di Roma, III. L'età Tardo Antica (613-642). Torino: Einaudi.

" Treitinger, O. (1956). Die oströmische Kaiser und Reichsidee nach ihrer Gestalt im höfischen Zeremoniell vom oströmischen Staats und Reichsgedanken. Darmstdt: Gentner Verlag. 